INPLASY

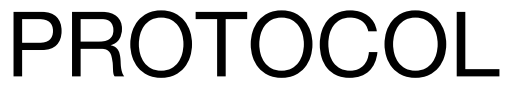

To cite: Liu et al. Meta analysis on Influencing Factors of evidence-based nursing ability of clinical nurses. Inplasy protocol 202130007. doi: 10.37766/inplasy2021.3.0007

Received: 02 March 2021

Published: 03 March 2021

Corresponding author: Qianqiu Liu

Iqq1995325@163.com

Author Affiliation:

Members of School of nursing, Henan University of Chinese medicine.

Support: Nothing.

Review Stage at time of this submission: Data analysis.

Conflicts of interest:

None declared.

\section{Meta analysis on Influencing Factors of evidence-based nursing ability of clinical nurses}

\author{
Liu, Q1; Jiang, G2; Ning, J3; Zhang, Y4.
}

Condition being studied: 1.The research team has systematically mastered the literature retrieval methods and meta-analysis methods. 2.There are many websites in the library resources of the school, which can comprehensively search the literature. 3.We have evidence-based instructors who can guide the research.

Eligibility criteria: Inclusive criteria: (1) the research type was a cross-sectional survey of evidence-based nursing ability of clinical nurses, and the subject was related to the analysis of influencing factors of evidence-based nursing ability of clinical nurses; (2) the research object was clinical front-line nurses; (3) the evaluation tool of evidence-based nursing ability was a relevant scale or questionnaire with effective reliability and validity verification, and the literature could extract data. Exclusion criteria: (1) repeated publication; (2) only abstracts without full text, data can not be extracted; (3) conference abstracts, news reports, reviews, expert comments, case reports and other literature.

INPLASY registration number: This protocol was registered with the International Platform of Registered Systematic Review and Meta-Analysis Protocols (INPLASY) on 03 March 2021 and was last updated on 03 March 2021 (registration number INPLASY202130007).

\section{INTRODUCTION}

Review question: Nothing.

Condition being studied: 1 . The research team has systematically mastered the literature retrieval methods and meta- analysis methods. 2.There are many websites in the library resources of the school, which can comprehensively search the literature. 3.We have evidence-based instructors who can guide the research.

Rationale: Meta analysis. 


\section{METHODS}

Search strategy: retrieval word: (日)clinical nurse, clinical nurses, nurse, nurses, (月) competence in evidence-based nursing practice, evidence-based ability, evidencebased nursing ability, evidence-based nursing (EBN), evidence-based, Evidencebased Nursing Practice, evidence-based practice(EBP). Database: PubMed, Embase, ScienceDirect, Web of Science, CNKI, WanFang Date, VIP, CBM Database.

\section{Study designs to be included: Cross} sectional study.

Eligibility criteria: Inclusive criteria: (1) the research type was a cross-sectional survey of evidence-based nursing ability of clinical nurses, and the subject was related to the analysis of influencing factors of evidencebased nursing ability of clinical nurses; (2) the research object was clinical front-line nurses; (3) the evaluation tool of evidencebased nursing ability was a relevant scale or questionnaire with effective reliability and validity verification, and the literature could extract data. Exclusion criteria: (1) repeated publication; (2) only abstracts without full text, data can not be extracted; (3) conference abstracts, news reports, reviews, expert comments, case reports and other literature.

Information sources: Electronic database.

Main outcome(s): Age, education background, professional title, position, professional attitude, literature reading, knowledge of evidence-based nursing, awareness of evidence-based nursing, evidence-based training and education of evidence-based medicine were the main influencing factors of evidence-based nursing ability of clinical nurses.

\section{Additional outcome(s): Nothing.}

Data management: Set up the document information extraction table, the main extracted information includes: basic information of literature, evaluation tools and contents, factors influencing nurses' evidence-based nursing ability, etc.The data were input into Revman software for calculation. 1.Comprehensive search of the database. 2. Two people screened the literature together, and the third person judged the conflict of the results. 3.The quality of the included articles was evaluated, only high quality literatures were included.

Quality assessment / Risk of bias analysis: Evaluation criteria for cross-sectional studies recommended by Agency for Healthcare Research and Quality.

Strategy of data synthesis: The variables were Standardized mean difference (SMD), SMD was used to transform the variables into $\log$ (or), and $95 \% \mathrm{Cl}$ was used for interval estimation. When $\mathrm{p}>0.1$ and $\mathrm{I} 2<$ $50 \%$, there was slight statistical heterogeneity between studies. Fixed effect model (FE) was used for analysis, otherwise random effect model (RE) was used for analysis.

Subgroup analysis: Influencing factors of evidence-based nursing ability.

Sensitivity analysis: RE and FE were used to calculate and compare the effects of these risk factors.

\section{Language: English.}

\section{Country(ies) involved: China.}

Keywords: clinical nurses; evidence based nursing ability; influencing factors; Meta analysis.

Contributions of each author:

Author 1 - Qianqiu Liu - The author drafted the first draft of the article, carried out the screening literature, data analysis and result analysis.

Email: lqq1995325@163.com

Author 2 - Guanhua Jiang - The author carried out screening literature and data analysis.

Email: 1226650459@qq.com

Author 3 - Jing Ning - The author carried out screening literature and data analysis. Email: 3119541982@qq.com 
Author 4 - Yongqin Zhang - The author read, provided feedback and approved the final manuscript.

Email: zhangyongqin168@163.com 